\title{
ORIGINAL
}

\section{Academic investigators' interest in promoting specified clinical trials : Questionnaire survey before and after implementation of the Clinical Trial Act}

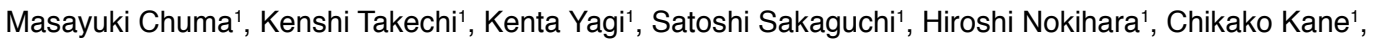 \\ Yasutaka Sato', Takahiro Niimura ${ }^{2}$, Mitsuhiro Goda ${ }^{3}$, Yoshito Zamami ${ }^{2,3}$, Keisuke Ishizawa ${ }^{2,3}$, and Hiroaki Yanagawa ${ }^{1}$ \\ ${ }^{1}$ Clinical Research Center for Developmental Therapeutics, Tokushima University Hospital, Tokushima, Japan, ${ }^{2}$ Department of Clinical Phar- \\ macology and Therapeutics, Tokushima University Graduate School of Biomedical Sciences, Tokushima, Japan, ${ }^{3}$ Department of Pharmacy, \\ Tokushima University Hospital, Tokushima, Japan
}

\begin{abstract}
Background : Enforcement of the 2018 Clinical Trials Act (CTA) in Japan resulted in strict and complicated regulations surrounding intervention studies. Few Japan-specific measures have been developed to promote intervention studies in Japan despite concerns about CTA's negative influence on such studies. Therefore, this study examined the changes in academic investigators' interest in conducting clinical studies before and after enforcement of the CTA to determine measures to promote specified clinical trials. Methods : We conducted a questionnaire survey with investigators belonging to the Institute of Biomedical Sciences, Tokushima University Graduate School, before and after enforcement of the CTA. Results : Investigators had lesser interest in intervention studies in the post-questionnaire survey (post) group than in the pre-questionnaire survey (pre) group. Their desire for "project management" was significantly higher in the post-group than in the pre-group. Their desire for "support for preparing documents when conducting specified clinical trials" was significantly higher in the group interested in conducting specified clinical trials than that in the not-interested group. Conclusion: We revealed that investigators were highly interested in "project management" and "support for preparing documents when conducting specified clinical trials" after enforcement of the CTA. Measures for these desires may promote specified clinical trials. J. Med. Invest. 68:71-75, February, 2021
\end{abstract}

Keywords : Clinical Trials Act, specified clinical trials, questionnaire survey, academic research organization

\section{INTRODUCTION}

In April 2018, the Clinical Trials Act (CTA) was enforced in Japan as a regulation for cases of misconduct, such as the Valsartan case wherein scientific misconduct and inappropriate relationship between academia and companies were identified (1). The Valsartan case involved the arrest of an employee of a company that marketed Valsartan by manipulating data from clinical trials conducted by medical and research institutions. The CTA aims to prevent such cases of scientific misconduct by enforcing improvements or discontinuation of clinical trials, which is otherwise not possible under the "Ethical Guidelines for Medical and Health Research Involving Human Subjects" in Japan (1-3). When investigators conduct clinical trials to clarify the efficacy or safety of pharmaceuticals, medical devices, or regenerative products ("pharmaceuticals," hereafter) in humans, such trials are subject to CTA (3). If they use unapproved/ off-label pharmaceuticals or receive research funding from a manufacturer with marketing approval for pharmaceuticals, the clinical trial is categorized as a specified clinical trial. Intervention studies, which account for most of the clinical studies, are essential to develop medicine and thus must be promoted under the CTA. Nakamura reported that there have been concerns about negative influences of the CTA's strict and complicated regulations on intervention studies that reduce clinical trial activity in

Received for publication May 31, 2020 ; accepted November 7, 2020.

Address correspondence and reprint requests to Masayuki Chuma, Clinical Research Center for Developmental Therapeutics, Tokushima University Hospital, 2-50-1 Kuramoto-cho, Tokushima 770-8503, Japan and Fax : +81-88-633-9295.
Japan (4). Moreover, in Japan, fewer intervention studies may be conducted due to the CTA in the future (4). Previous studies show that the number of clinical trials decreased remarkably when the EU directive was introduced in $2004(5,6)$.

The CTA regulations are similar to the Good Clinical Practice standards of the International Conference on Harmonisation of Technical Requirements for Registration of Pharmaceuticals for Human Use, which are the regulatory cornerstone of CTA. Nevertheless, there is a need for studies that consider Japan-specific issues-such as the certified review board (CRB)'s review fee, conflict of interest management plan, and registration at the Japan Registry of Clinical Trials (jRCT ; a newly established clinical trial registry website) — for promoting clinical studies (1, 3). Moreover, which Japan-specific measures are necessary to promote specified clinical trials that must be conducted under the CTA remains unknown.

In Japan, the main role of the academic research organizations (AROs) is promoting clinical research. AROs with full functions that provide a sufficient management system for performing clinical study and promote clinical trials under regulation of Good Clinical Practice are approved as core clinical trial hospitals by the Ministry of Health, Labour and Welfare (7). However, there are 13 organizations that have an ARO with full functions (as of April 1, 2020) ; they represent only $0.16 \%$ $(13 / 8255)$ of the total 8,255 hospitals in Japan, of which 11 are university hospitals $(8,9)$. There are also $98 \mathrm{CRBs}$, and 68 of them are located at universities/university hospitals. Thus, since most universities/university hospitals with CRBs (83.8\%, 57/68) have AROs without full functions, it is important to examine measures that can promote clinical research among researchers at these organizations in order to facilitate specified clinical trials. The aim of this study was to evaluate the desire 
and interests of investigators at The Tokushima University (one of the institutions with CRBs but AROs without full functions) regarding the support of clinical research and to determine the impact of CTA implementation at an institution with inadequate support for clinical research.

\section{METHODS}

Questionnaire distribution and interests identified through the survey

We utilized a questionnaire survey to examine the changes in the interest in conducting clinical studies before and after CTA's implementations. The survey was conducted with investigators belonging to the Institute of Biomedical Sciences, Tokushima University Graduate School, because this institution does not have an ARO but has a CRB. Five questionnaire sheets were distributed to all departments before and after enforcement of CTA (pre-group : pre-questionnaire survey, 30 departments ; post-group : post-questionnaire survey, 33 departments), wherein at least five full-time physicians and dentists who mainly conduct intervention studies at Tokushima University are enrolled (pre-group : total 150 sheets ; post-group : total 165 sheets). Four faculty members were consulted to obtain details about the respondents' interest in promoting specified clinical trials, and we asked academic investigators who usually conduct clinical trials and clinical research to answer the questionnaires as much as possible. The questionnaire asked about the investigators' characteristics such as gender, position, experience with clinical trials, ethics committee applications, interest in each study design, and desire for human and physical resources required for the smooth promotion of clinical research. Many research organizations with AROs without full functions have evolved to support industry-initiated clinical trials, but are presumably not well-equipped to promote academia-initiated clinical research by their investigators $(10,11)$. Since this feature is considered to be common to research organizations without
AROs with full functions, the desires and interests considered necessary to promote research in these organizations were determined for various occupations (physician, pharmacist, nurse, and nutritionist) and departments.

We arranged for biostatisticians and faculty members (current faculty members : two physicians and two pharmacists) and focused on educational opportunities across the pre- and post-questionnaire surveys (Figure 1). The pre-questionnaire survey assessed interest/desire for opportunities for education when conducting a clinical study. The post-questionnaire survey identified two interests-(1) support for preparing documents when conducting specified clinical trials and (2) establishment of a fund for conducting specified clinical trials. This is because these interests were required to support application documents and obtain funding after the CTA. The pre- and post-questionnaire surveys both identified six common interests-(1) project management, (2) academic office for promoting clinical study, (3) support for conducting clinical study, (4) data management, (5) electronic data capture, and (6) quality control.

\section{Statistical analysis}

Data were presented as rates and percentages of the respondents. The $p$-value of $<0.05$ was considered to be statistically significant.

\section{RESULTS}

\section{Characteristics of the respondents}

The response rate for the pre-questionnaire survey was $70.7 \%$ (106/150) and that for the post-questionnaire survey was $75.2 \%$ $(124 / 165)$; there was no significant difference in the two groups (Table 1). In these two groups, most respondents were at the position of non-professor (pre-group : $81.1 \%$, post-group : 76.6\%), followed by professor (pre-group : $15.1 \%$, post-group : $15.3 \%$ ). In both groups, more than $90 \%$ of respondents were research faculty members and had experience of clinical trials or application

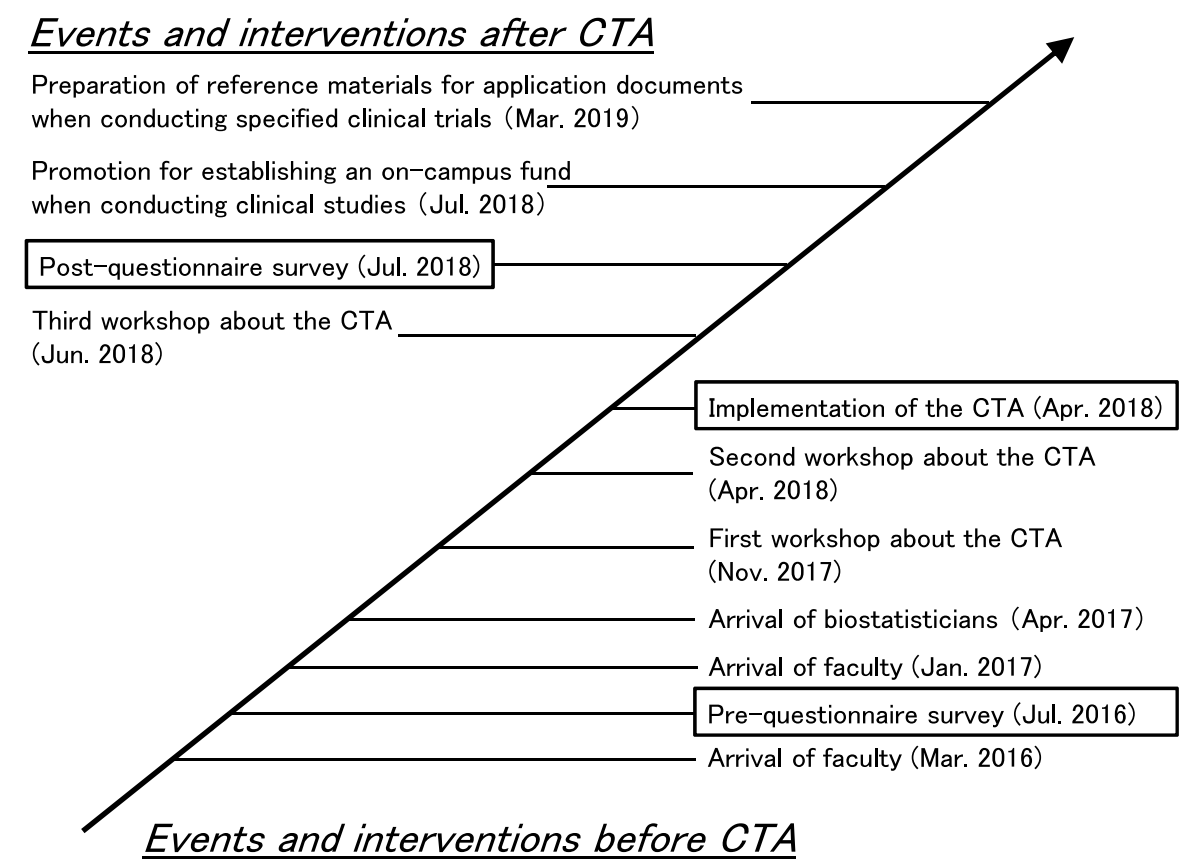

Figure 1. Events and interventions to promote clinical studies before and after implementation of the Clinical Trials Act (CTA) 
Table 1. Comparison of investigators' interest in conducting clinical studies before and after implementation of the Clinical Trials Act (CTA). Data are presented based on the number of respondents (rates and percentages).

\begin{tabular}{|c|c|c|c|}
\hline & \multicolumn{2}{|c|}{ Questionnaire survey } & \multirow{2}{*}{$P$ value } \\
\hline & pre $(n=106)$ & post $(n=124)$ & \\
\hline Response rate (\%) & 70.7 & 75.2 & 0.377 \\
\hline Gender (male/female) & $92 / 14$ & $98 / 26$ & 0.162 \\
\hline Position & & & 0.376 \\
\hline Director/professor & $16(15.1)$ & $19(15.3)$ & \\
\hline Faculty other than professor & $86(81.1)$ & $95(76.6)$ & \\
\hline Medical staff & $1(0.94)$ & $6(4.84)$ & \\
\hline Graduate student & $1(0.94)$ & $3(2.42)$ & \\
\hline Other & $2(1.89)$ & $1(0.81)$ & \\
\hline Experience of performing clinical trial & $72(67.9)$ & $71(57.3)$ & 0.104 \\
\hline Experience of application to an ethics committee & $91(85.9)$ & $107(86.3)$ & 0.104 \\
\hline \multicolumn{4}{|l|}{ Interest in clinical study } \\
\hline Intervention study & $53(50.0)$ & $43(34.7)$ & 0.023 \\
\hline Observational study & $68(64.2)$ & $92(74.2)$ & 0.115 \\
\hline Neither & $0(0.0)$ & $1(0.81)$ & 1.000 \\
\hline \multicolumn{4}{|l|}{ Desire for a support system for conducting clinical study } \\
\hline Project management & $55(51.9)$ & $89(71.8)$ & 0.003 \\
\hline Academic office for promoting clinical study & $53(50.0)$ & $77(62.1)$ & 0.083 \\
\hline Support for conducting clinical study (e.g., informed consent and schedule management) & $71(67.0)$ & $93(75.0)$ & 0.191 \\
\hline Data management & $77(72.6)$ & $86(69.4)$ & 0.663 \\
\hline Electronic data capture & $56(52.8)$ & $70(56.5)$ & 0.597 \\
\hline Quality control (e.g., monitoring and audit) & $52(49.1)$ & $70(56.5)$ & 0.29 \\
\hline Opportunity for education when conducting a clinical study & $49(46.2)$ & - & \\
\hline Support for preparing documents when conducting specified clinical trials & & $107(86.3)$ & \\
\hline Establishment of a fund in conducting specified clinical trials & & $99(79.8)$ & \\
\hline
\end{tabular}

to an ethics committee. Thus, investigators who usually conduct clinical studies and clinical trials accounted for the majority of respondents, suggesting that we obtained the response from investigators that we expected. In the post-group, the interest in intervention study was significantly lower than that in the pregroup (pre-group : $50.0 \%$, post-group : $34.7 \%$ ), although there was no significant difference between the two groups in experience of clinical trials, application to an ethics committee, and interest in observational study. This suggests that investigators' interest in intervention studies decreased after enforcement of the CTA.

Comparison of investigators' interest in conducting clinical studies before and after enforcement of the CTA

A comparison of the six interests common to both groups showed that investigators' interest in "project management" in the post-questionnaire survey was significantly higher than that in the pre-questionnaire survey (pre : $51.9 \%$, post : $71.8 \%$ ), suggesting that their interest in conducting clinical studies was increasing. As expected, the investigators responded highly ( $\geq 70 \%$ ) to "support for preparing documents when conducting specified clinical trials" (86.3\%) and "establishment of a fund when conducting specified clinical trials" (79.8\%), which were added in the post-questionnaire survey. Similarly, there was high interest in "support for conducting a clinical study" (75.0\%) and "project management" (71.8\%).
Comparison of investigators' interest in conducting specified clinical trials

We compared the desire for conducting clinical studies among investigators who were interested in conducting specified clinical trials (interested group : 63) and those who were not (not-interested group : 61). This is because the post-group's interest in intervention studies was significantly lower than in the pregroup (Table 2). Approximately 50\% (63/124) of respondents (interested group) desired to conduct specified clinical trials. Moreover, the interested group's rate of interest in intervention studies (46\%) was significantly higher than that of the not-interested group (23\%). This suggests that the interest in specified clinical trials is related to an interest in intervention studies. The interested group favored "support for preparing documents when conducting specified clinical trials" (96.8\%) significantly more than the not-interested group (75.4\%). Moreover, more than $70 \%$ of respondents in both groups were generally interested in "establishment of a fund when conducting specified clinical trials," "support for conducting a clinical study," and "support for preparing documents when conducting specified clinical trials."

Comparison of investigators' interest in conducting specified clinical trials between participants and non-participants groups

We classified the pre-group into two groups-participation group and non-participation group - to examine investigators' 
interest, because participation in workshops on CTA is likely to affect the interests/desire of the investigators. Interest in conducting intervention study among workshop participants was higher than that among non-participants. On the other hand, the eight items related to supporting performing clinical research were not significantly different between the two groups (Table 3).

\section{DISCUSSION}

In Japan, there is a concern that implementation of the CTA in 2018 may reduce the number of intervention studies conducted. Therefore, unique measures that promote specified clinical trials to support investigators are required, because the CTA is different from international regulations. In this study, we conducted a questionnaire survey before and after enforcement of the CTA to understand the investigators' interest in conducting specified clinical trials and to determine the basis of measures to promote specified clinical trials. Among the four items that investigators were highly interested in after enforcement of the CTA- "project management," "support for conducting a clinical study," "support for preparing documents when conducting specified clinical trials," and "establishment of a fund when conducting specified clinical trials"-investigators' interest in "project management" in the post-group was significantly higher than that in the pregroup. Similarly, among the three items in which investigators of both interested and not-interested groups classified from the post-group were highly interested in-"establishment of a fund when conducting specified clinical trials," "support for conducting a clinical study," and "support for preparing documents when conducting specified clinical trials-the interest in "support for preparing documents when conducting specified clinical trials" was significantly higher in the interested group than in the not-interested group. A previous report showed that in Denmark, where support organizations provide free assistance to academic clinical researchers to promote clinical research, although the number of clinical trials declined after implementation of the 2004 European Clinical Trials Directive, this decline ended and then the number of clinical trials increased (12). This suggests that development of a support organization for clinical trials is necessary to promote interventional studies (12). In fact, it is necessary to create a seamless department in each organization that is led by investigators who have experience in designing and publishing clinical trials (13).

Moreover, we have previously reported the necessity to develop new guidelines for intervention trials (14) and to provide support to investigators conducting clinical studies under awareness of

Table 2. Comparison of investigators' interest in conducting specified clinical trials. Data are presented as number of respondents (rates ; percent).

\begin{tabular}{|c|c|c|c|}
\hline & \multicolumn{2}{|c|}{ Performing "specified clinical trials" } & \multirow{2}{*}{$P$ value } \\
\hline & Interested $(n=63)$ & Not interested $(n=61)$ & \\
\hline Interest in intervention study & $29(46.0)$ & $14(23.0)$ & 0.008 \\
\hline \multicolumn{4}{|l|}{ Desire for a support system for conducting clinical study } \\
\hline Project management & $48(76.2)$ & $41(67.2)$ & 0.320 \\
\hline Academic office for promoting clinical study & $42(66.7)$ & $35(57.4)$ & 0.355 \\
\hline Support for conducting clinical study & $47(74.6)$ & $46(75.4)$ & 1.000 \\
\hline Data management & $43(68.3)$ & $43(70.5)$ & 0.847 \\
\hline Electronic data capture & $38(60.3)$ & $32(52.5)$ & 0.469 \\
\hline Quality control & $39(61.9)$ & $31(50.8)$ & 0.277 \\
\hline Support for preparing documents when conducting specified clinical trials & $61(96.8)$ & $46(75.4)$ & $<0.001$ \\
\hline Establishment of a fund when conducting specified clinical trials & $53(84.1)$ & $46(75.4)$ & 0.267 \\
\hline
\end{tabular}

Table 3. Comparison of investigators' interest in conducting specified clinical trials between participant and non-participant groups. Data are presented as number of respondents (rates ; percent)

\begin{tabular}{lccc}
\hline & \multicolumn{2}{c}{ Participation in CTA workshop } & $P$ value \\
& Yes (n=41) & No (n=83) & \\
\hline Interest in conducting intervention study & $20(48.8)$ & $23(27.7)$ & \\
Desire for a support system for conducting clinical study & & & \\
$\quad$ Project management & $29(70.7)$ & $60(72.3)$ & 1.000 \\
Academic office for promoting clinical study & $26(63.4)$ & $51(61.5)$ & 1.000 \\
Support for conducting clinical study & $34(82.9)$ & $59(71.1)$ & 0.189 \\
Data management & $28(68.3)$ & $58(69.9)$ & 1.000 \\
Electronic data capture & $25(61.0)$ & $45(54.2)$ & 0.565 \\
Quality control & $26(63.4)$ & $44(53.0)$ & 0.337 \\
Support for preparing documents when conducting specified clinical trials & $34(82.9)$ & $73(88.0)$ & 0.580 \\
Establishment of a fund when conducting specified clinical trials & $32(78.1)$ & $67(80.7)$ & 0.813 \\
\hline
\end{tabular}


the CTA through the following : arranging faculty members and biostatisticians, creating opportunities for education, promoting the establishment of an on-campus fund for conducting clinical studies, and preparing reference material for application documents when conducting specified clinical trials before enforcement of the CTA (Figure 1) (11). We also provided support for the preparation of documents and consultation for designing clinical research such as through intervention and observational studies by making available four faculty members (two physicians and two pharmacists) before enforcement of the CTA. Results of the pre- and post-questionnaire surveys revealed that investigators highly desire "project management" and "support for preparing documents when conducting specified clinical trials." Clinical Research Center for Developmental Therapeutics, Tokushima University Hospital has already begun supporting investigators belong to its research institution by providing "support for preparing documents when conducting specified clinical trials." This is expected to contribute to the promotion of specified clinical trials, as these measures are based on the desires and interests identified in this study in addition to the previous efforts of preparing documents and providing consultation for the design of clinical research before enforcement of the CTA. In contrast, there were no significant differences in the level of desire between participants and non-participants in the workshop. Because the workshop was held immediately before and after the enforcement of the CTA, it was mainly focused on the overview and regulations of the CTA and was unable to focus on the promotion measures of specified clinical trials, which may have been one of the reasons. Since researchers interested in intervention study participated in the workshop, continued workshops with contents related to the promotion of clinical research would increase researchers' understanding and desire for support of clinical research. Measures based on the interests of investigators belonging to research organizations that do not have AROs with full functions (which account for most organizations in Japan) may lead to the development of a universal system to promote specified clinical trials.

There are two limitations in this study. First, it conducted a questionnaire survey at a single organization. Inter-facility bias and excess or deficiency of the questionnaire's survey items may affect the results. Second, biostatisticians and faculty members to promote clinical research were arranged across the pre- and post-questionnaire surveys, which may have influenced researchers' desire for conducting clinical study in the questionnaire survey.

\section{CONCLUSION}

This study revealed that academic investigators are highly desirous of project management and support for drafting and editing the protocol after enforcement of the CTA. Measures that address these desires may promote specified clinical trials for intervention studies, in addition to the measures identified by previous studies.

\section{CONFLICT OF INTERESTS}

None

\section{ACKNOWLEDGEMENTS}

We would like to thank Editage (Editage, Tokyo, Japan) for English-language editing.

\section{FUNDING}

None

\section{REFERENCES}

1. Ministry of Health, Labour and Welfare. Clinical Trials Act [Internet] ; 2017 [cited 2019Mar21].https://www.mhlw.go.jp/ file/06-Seisakujouhou-10800000-Iseikyoku/0000213334. pdf (in Japanese)

2. MuCurry $\mathrm{J}$ : Former Novartis employee arrested over Valsartan data. Lancet $383: 2111,2014$

3. Japan Registry of Clinical Trials. Certified review board information service [Internet] ; n.d. [cited 2019 Mar 21]. https://jrct.niph.go.jp/ (in Japanese)

4. Nakamura K, Shibata T : Regulatory Changes After the Enforcement of the New Clinical Trials Act in Japan. Jpn J Clin Oncol 50 : 399-404, 2020

5. European Commission. Impact assessment on the revision of the "Clinical Trials Directive" 2001/20/EC [Internet] ; n.d. [cited 2019 Mar 21]. https://ec.europa. eu/health/sites/health/files/files/clinicaltrials/2012_07/ impact_assessment_part1_en.pdf

6. Watson R : EU nations approve law to overhaul clinical trials. BMJ 347 : f7682, 2013

7. Fukushima M, Austin C, Sato N, Maruyama T, Navarro E, Rocca M, Demotes J, Haendel M, Volchenboum SL, Cowperthwaite M, Silverstein JC, Webb C, Sim I, Chase M, Speakman J, Augustine E, Ford DE, Kush R: The global academic research organization network : Data sharing to cure diseases and enable learning health systems. Learn Health Syst 3 : e10073, 2018

8. Ministry of Health, Labour and Welfare. Clinical research core centers [Internet] ; n.d. [cited 2020 Apr 1]. https://www. mhlw.go.jp/stf/seisakunitsuite/bunya/tyukaku.html (in Japanese)

9. Ministry of Health, Labour and Welfare. Clinical research core centers [Internet] ; n.d. [cited 2020 Sep 9]. https://www. mhlw.go.jp/toukei/list/79-1a.html (in Japanese)

10. Yanagawa H, Nohihara H, Yokoi H, Houchi H, Nagai M, Yamashita R, Suganuma N, Hyodo M, the Shikoku Collaborative Group for Promotion of Clinical Trials : Present status and perspectives on future roles of Japanese clinical research coordinators. J Clin Med Res 10 : 877-882, 2018

11. Yanagawa $\mathrm{H}$, Katashima $\mathrm{R}$, Takeda $\mathrm{N}$ : Research ethics committees in Japan: A perspective from thirty years of experience at Tokushima University. J Med Invest 62 : 114118,2015

12. Berendt L, Håkansson C, Bach KF, Dalhoff K, Andreasen PB, Petersen LG, Andersen E, Poulsen HE : Effect of European clinical trials directive on academic drug trials in Denmark : Retrospective study of applications to the Danish Medicines Agency 1993-2006. BMJ 336 : 33-35, 2008

13. Stewart PM, Stears A, Tomlinson JW, Brown MJ : Regulation : The real threat to clinical research. BMJ 337 : a1732, 2008

14. Yanagawa $\mathrm{H}$ : Current regulatory systems for clinical trials in Japan : Still room for improvement. Clin Res Regul Aff $31: 25-28,2014$ 